\title{
Pulmonary rehabilitation outcome of exercise-induced oxygen desaturation in systemic sclerosis with interstitial lung disease
}

\author{
Fujiko Someya $^{1^{*}}$, Naoki Mugii $^{2}$ \\ ${ }^{1}$ School of Health Sciences, Kanazawa University, Kanazawa, Japan; *Corresponding Author: fujiko@mhs.mp.kanazawa-u.ac.jp \\ ${ }^{2}$ Division of Rehabilitation, Kanazawa University Hospital, Kanazawa, Japan \\ Received 1 March 2013; revised 16 April 2013; accepted 15 May 2013 \\ Copyright (C) 2013 Fujiko Someya, Naoki Mugii. This is an open access article distributed under the Creative Commons Attribution \\ License, which permits unrestricted use, distribution, and reproduction in any medium, provided the original work is properly cited.
}

\begin{abstract}
While exercise capacity in systemic sclerosis with interstitial lung disease could be improved by exercise training, the training outcome of exercise-induced oxygen desaturation has not been examined. The aim of this study was to investigate the effect of pulmonary rehabilitation on exercise-induced oxygen desaturation during the six-minute walk test and to detect the factors affecting outcome retrospectively. Patients showing impaired exercise capacity ( $\leq 80 \%$ of predicted) and/or exercise-induced oxygen desaturation $\left(\leq-4 \%\right.$ in $\left.\mathrm{SpO}_{2}\right)$ at the end of the six-minute walk test underwent routine walking exercise. Sixteen patients with stable systemic sclerosis completed exercise training for 55 days on average. The mean six-minute walk distance improved from $467 \mathrm{~m}$ to $502 \mathrm{~m}(P=0.0012)$. The improvement in distance was negatively related to baseline distance $\left(R^{2}=0.28, P=0.037\right)$, but was not related to parameters from pulmonary function tests and echocardiograms. Oxygen saturation was normal at rest, but was decreased in fifteen patients at the end of the test. Exercise-induced oxygen desaturation was positively related to the diffusion capacity of the lungs for carbon monoxide at baseline $\left(R^{2}=0.33\right.$, $P=0.026$ ); however, it was not related to any cardiopulmonary parameters after intervention. Seven of sixteen patients ameliorated exerciseinduced oxygen desaturation or showed no oxygen desaturation after exercise training, while others deteriorated. No cardiopulmonary parameters affected the training outcome of exercise-induced oxygen desaturation. Exercise training was beneficial in improving exercise toler-
\end{abstract}

ance, but training effects and mechanisms on exercise-induced oxygen desaturation still need more studies to be explained.

Keywords: Systemic Sclerosis; Interstitial Lung Disease; Training; Oxygen Saturation; Exercise Capacity

\section{INTRODUCTION}

Impaired exercise capacity in systemic sclerosis was suggested to occur due to multiple organ dysfunctions [1], and could be improved by training exercise [2]. Interstitial lung disease and cardiac diastolic disturbances in systemic sclerosis were reported to be factors relating to reduced exercise capacity and exercise-induced oxygen desaturation [3-7]. The benefit of exercise training was more pronounced in interstitial lung disease patients with poor exercise intolerance [8]; however, the effect of exercise training on exercise-induced oxygen desaturation has not been examined. A case report on a systemic sclerosis patient showed reduced hypoxia risk by long-term exercise training without improvements in exercise capacity [9]. This suggested that exercise training may possibly affect exercise intolerance and exercise-induced oxygen desaturation independently.

The purpose of this study was to investigate retrospectively the training outcomes of exercise capacity and exercise-induced oxygen desaturation in patients at the stable period of systemic sclerosis with interstitial lung disease. Subsequently, we examined outcomes in relation to cardiopulmonary parameters.

\section{METHODS}

\subsection{Subjects and Methods}

Ninety-four consecutive stable adult systemic sclerosis 
patients with interstitial lung disease transferred to the Division of Rehabilitation Medicine of our university hospital for the six-minute walk test (6MWT) between 2008 and 2011. Interstitial lung disease was diagnosed by clinical symptoms, high resolution CT and/or open lung biopsy. Exclusion criteria for 6MWT were foot pain with or without digit ulcers, back pain, severe cough, and muscle weakness to walk; therefore 75 of them underwent 6MWT. Forty-one patients showed exercise intolerance, $\leq 80 \%$ in $6 \mathrm{MWT}$ distance of predicted, and/or exercise-induced oxygen desaturation $\left(\leq-4 \%\right.$ in $\left.\mathrm{SpO}_{2}\right)$ at the end of 6MWT. Twenty-five patients of them visited for single evaluation of 6MWT. Remained 16 patients (10 females and 6 males, mean age $55.4 \pm 12.3$ years) underwent exercise training five days a week under the supervision of a therapist as routine pulmonary rehabilitation treatment, setting the lowest $\mathrm{SpO}_{2}$ during exercise training as $90 \%$. All of them could complete the walking exercise for 55 days on average. None had received pulmonary rehabilitation before that. Thirteen of them were diagnosed as diffuse cutaneous types and three were limited cutaneous types. Anti-topoisomerase-I antibodies were found in 10 patients, anti-RNAP antibodies in three, other autoantibodies in two, and one patient was negative.

The distance of 6MWT was calculated to a percent of the predicted value by the Enright formula [10] taking gender, age, height, and weight into consideration. $\mathrm{SpO}_{2}$ was monitored by a pulse oximeter with a sensor on the forehead to avoid evaluation errors by poor circulation on the fingertip [11]. $\mathrm{SpO}_{2}$ was above $96 \%$ without oxygen supplement at rest and a reduction in the percent of $\mathrm{SpO}_{2}$ at the end of $6 \mathrm{MWT}$ was defined as $\Delta \mathrm{SpO}_{2}\left(\Delta \mathrm{SpO}_{2}\right.$ $=\mathrm{SpO}_{2}$ at end of $6 \mathrm{MWT}-\mathrm{SpO}_{2}$ at rest).

As for clinical parameters, we collected data of percent predicted of vital capacity (VC) and diffusion capacity of the lungs for carbon monoxide (DLCO) from spirometry. Left ventricular ejection fraction (EF), early diastolic filling peak velocity/late diastolic filling peak velocity ratio $(E / A)$, early diastolic filling peak velocity/early diastolic mitral annular velocity ratio $\left(E / E^{\prime}\right)$, and right ventricular systolic pressure estimated from tricuspid regurgitating velocity (RVsystP) were measured with echocardiography. This study was approved by the Ethics Committee of the University and all patients gave their written informed consent to participate in the study.

\subsection{Statistics}

Simple linear regression between 6MWT distance or $\Delta \mathrm{SpO}_{2}$ and clinical parameters was performed. The patients were classified into the amelioration and deterioration groups according to outcomes of $\Delta \mathrm{SpO}_{2}$ after exercise training. The Student t-test was used to compare clinical parameters between the two groups of patients.
Statistical analyses were performed with JMP8.0 (SAS Institute, Cary, NC). In all analyses, $P<0.05$ was taken to indicate significance.

\section{RESULTS}

Improvements in mean 6MWT distance were significant, from $467 \mathrm{~m}$ to $502 \mathrm{~m}(P=0.0012)$ or from $82 \%$ to $88 \%$ of predicted $(P=0.0011)$ after exercise training, but one patient failed to elongate walking distance (Figure 1). A significant negative correlation between improvements in percent predicted of 6MWT distance and baseline 6MWT distances $(P=0.037)$ was shown (Table 1); however, there was no correlation between the improvements and baseline cardiopulmonary parameters.

Exercise-induced oxygen desaturation was resulted in amelioration or the same in seven patients and in deterioration in nine patients after exercise training (Figure 1). No difference was found in cardiopulmonary parameters and baseline status regarding 6MWT distance or $\Delta \mathrm{SpO}_{2}$ between outcomes of exercise-induced oxygen desaturation (Table 2). Baseline $\Delta \mathrm{SpO}_{2}$ was positively related to DLCO $\left(R_{2}=0.33, P=0.026\right)$, but there was no correlated parameter with $\Delta \mathrm{SpO}_{2}$ after exercise training (Table 3).

\section{DISCUSSION}

We demonstrated elongation of 6MWT distance after walking training and it was more beneficial to patients with a lower exercise capacity at baseline, whereas no cardiopulmonary parameters were related to the improvement in 6MWT distance. Improvements in exercise

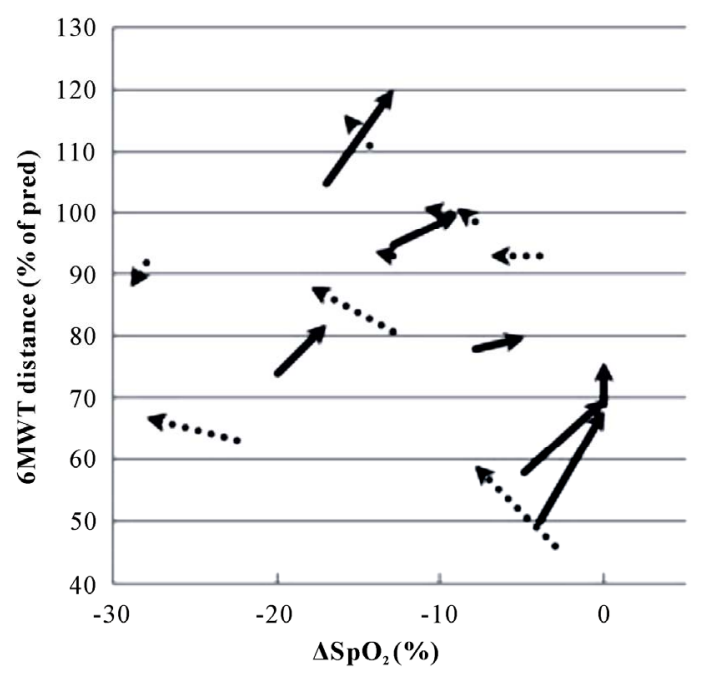

Figure 1. Changes in outcomes of $6 \mathrm{MWT}$ distance and $\Delta \mathrm{SpO}_{2}$ from baseline to post-training for all patients $(n=16)$. Fifteen patients improved their 6MWT distance. Arrows with solid lines represent ameliorations or no change in $\Delta \mathrm{SpO}_{2}(n=7)$, while arrows with broken lines represent deteriorations in $\Delta \mathrm{SpO}_{2}(n=9)$. 
Table 1. Relationship between improvements in 6MWT distance and clinical parameters.

\begin{tabular}{ccc}
\hline & $R^{2}$ & $P$ \\
\hline Age (yrs) & 0.013 & 0.67 \\
Duration of disease (yrs) & 0.060 & 0.37 \\
Baseline 6MWT distance (\% of pred) & 0.28 & $0.037^{*}$ \\
Baseline $\Delta \mathrm{SpO}_{2}(\%)$ & 0.12 & 0.19 \\
VC (\% of pred) & $<0.001$ & 0.96 \\
DLCO (\% of pred) & 0.045 & 0.45 \\
EF (\%) & 0.0046 & 0.80 \\
$E / A$ & 0.064 & 0.34 \\
$E / E^{\prime}$ & 0.0073 & 0.75 \\
RVsystP (mmHg) & 0.014 & 0.67 \\
\hline
\end{tabular}

$n=16 .{ }^{*} P<0.05$. 6MWT; the six-minute walk test: DLCO; diffusion capacity of the lungs for carbon monoxide: $E / A$; early diastolic filling peak velocity/late diastolic filling peak velocity ratio: $E / E^{\prime}$; early diastolic filling peak velocity/early diastolic mitral annular velocity ratio: EF; left ventricular ejection fraction: RVsystP; right ventricular systolic pressure: VC; vital capacity.

Table 2. Comparison of clinical parameters between outcomes of exercise-induced oxygen desaturation.

\begin{tabular}{cccc}
\hline & $\begin{array}{c}\text { Ameliorated } \\
(n=7)\end{array}$ & $\begin{array}{c}\text { Deteriorated } \\
(n=9)\end{array}$ & $P$ \\
\hline Age (yrs) & $55.0 \pm 11.2$ & $55.7 \pm 13.7$ & 0.91 \\
Duration of disease (yrs) & $4.8 \pm 5.4$ & $6.4 \pm 3.8$ & 0.50 \\
$\begin{array}{c}\text { Baseline 6MWT distance } \\
\text { (\% of pred) }\end{array}$ & $75.6 \pm 19.4$ & $86.3 \pm 20.1$ & 0.30 \\
Baseline $\Delta \mathrm{SpO}_{2}(\%)$ & $9.6 \pm 7.3$ & $12.7 \pm 8.1$ & 0.44 \\
$\mathrm{VC}(\%$ of pred) & $73.7 \pm 17.7$ & $78.4 \pm 21.5$ & 0.64 \\
$\mathrm{D}_{\mathrm{LCO}}(\%$ of pred) & $44.7 \pm 18.5$ & $45.9 \pm 12.5$ & 0.89 \\
$\mathrm{EF}(\%)$ & $73.7 \pm 3.1$ & $68.6 \pm 7.8$ & 0.10 \\
E/A & $1.0 \pm 0.4$ & $1.1 \pm 0.3$ & 0.73 \\
E/E' & $13.2 \pm 6.3$ & $8.7 \pm 1.8$ & 0.11 \\
$\mathrm{RV}$ systP (mmHg) & $31.0 \pm 11.0$ & $33.8 \pm 15.4$ & 0.68 \\
\hline
\end{tabular}

Values are mean \pm SD. No significant difference was found in parameters between outcomes. 6MWT; the six-minute walk test: DLCO; diffusion capacity of the lungs for carbon monoxide: $E / A$; early diastolic filling peak velocity/late diastolic filling peak velocity ratio: $E / E^{\prime}$; early diastolic filling peak velocity/early diastolic mitral annular velocity ratio: EF; left ventricular ejection fraction: RVsystP; right ventricular systolic pressure: $\mathrm{VC}$; vital capacity.

capacity by exercise training have been well documented in patients with systemic sclerosis [2]. In interstitial lung disease, it was also reported that age, gender, baseline forced vital capacity, baseline DLCO, smoking history, long-term oxygen therapy, and baseline Borg score were not predictors of the improvement in 6MWT distance, and impaired baseline 6MWT distance was the only significant predictor [8]. This implied that elongation of 6MWT distance after walking training would not be influenced by baseline cardiopulmonary function, but by
Table 3. Simple linear regression between exercise-induced oxygen desaturation and clinical parameters.

\begin{tabular}{ccccc}
\hline & \multicolumn{4}{c}{$\Delta \mathrm{SpO}_{2}(\%)$} \\
\cline { 2 - 5 } & \multicolumn{3}{c}{ Baseline } & \multicolumn{2}{c}{ After training } \\
\cline { 2 - 5 } & $R^{2}$ & $P$ & $R^{2}$ & $P$ \\
\hline VC (\% of pred) & 0.026 & 0.55 & 0.0084 & 0.74 \\
DLCO (\% of pred) & 0.33 & $0.026^{*}$ & 0.21 & 0.089 \\
EF (\%) & 0.0044 & 0.81 & 0.0059 & 0.78 \\
E/A & 0.077 & 0.30 & 0.054 & 0.39 \\
$E / E^{\prime}$ & $<0.001$ & 0.94 & 0.065 & 0.34 \\
RVsystP (mmHg) & 0.066 & 0.34 & 0.14 & 0.15 \\
\hline
\end{tabular}

$\mathrm{n}=16 .{ }^{*} \mathrm{P}<0.05$. $6 \mathrm{MWT}$; the six-minute walk test: DLCO; diffusion capacity of the lungs for carbon monoxide: $E / A$; early diastolic filling peak velocity/late diastolic filling peak velocity ratio: $E / E^{\prime}$; early diastolic filling peak velocity/early diastolic mitral annular velocity ratio: $\mathrm{EF}$; left ventricular ejection fraction: RVsystP; right ventricular systolic pressure: VC; vital capacity.

another factor such as muscle adaptation, which was one of the factors of exercise capacity [12].

Baseline $\Delta \mathrm{SpO}_{2}$ was affected by DLCO. Previously, $\Delta \mathrm{SpO}_{2} \leq-4 \%$ during $6 \mathrm{MWT}$ was found to be related to age, dyspnea index, positive anti-Scl-70 autoantibody, and forced vital capacity $<80 \%$ of predicted in systemic sclerosis; however, DLCO was not examined [4]. As DLCO reflects oxygen perfusion through the alveolar wall, interstitial lung lesions affect this parameter to reduce $\mathrm{SpO}_{2}$ in idiopathic interstitial pneumonia [13]. Thus, reductions in DLCO likely induced oxygen desaturation during exercise in systemic sclerosis.

A relationship between $\triangle \mathrm{SpO}_{2}$ and DLCO was not found after exercise training. Besides that, nine patients had more severe exercise-induced oxygen desaturation. This may be the result of an increase in oxygen consumption due to elongation of the walking distance. In contrast, an explanation for ameliorations in exerciseinduced oxygen desaturation should be discussed. Suitable increases in oxygen supply are necessary to compensate for increases in oxygen consumption, though patients received exercise training during the stable period of the disease and DLCO may not be expected to improve [14]. A previous study showing improvements in oxygen saturation after aerobic exercise speculated that this result was due to more efficient ventilatory mechanics, which led to better gaseous exchange [2]. As another possible explanation for oxygen saturation, cardiac function may have improved after exercise training.

Generally, $E / A$ and $E / E^{\prime}$ express left ventricular dilatation function, and $E / A$ is known to be reduced [15-17] and $E / E^{\prime}$ increased [18] in systemic sclerosis patients. Diastolic dysfunction was suggested to occur due to repeated focal ischemia leading to myocardial fibrosis in systemic sclerosis [19] and induced exercise 
intolerance [5]. Similarly, pulmonary arterial hypertension in systemic sclerosis has been reported in relation to exercise intolerance $[3,20]$ and mortality $[21]$ suggesting right ventricular diastolic dysfunction [22]. In this study, there was no difference between the ameliorated and deteriorated groups in baseline parameters from echocardiography. Whereas, cardiac parameters were not examined after exercise training, so closer examination regarding exercise-induced oxygen desaturation may be necessary in future studies.

Hypoxic states may be harmful for digit ulcers and myocardial ischemia due to microvascular lesions in systemic sclerosis $[19,23]$. We set the lowest $\mathrm{SpO}_{2}$ during exercise training as $90 \%$, so exercise was consequently directed to be interval training. Hence, patients spend their daily lives walking continuously for more than six minutes, which suggested that they were easily getting into hypoxic states even after exercise training. An indication of exercise training should be considered to avoid the risk of exercise-induced oxygen desaturation independently of improvements in exercise capacity. Arterial blood gas analysis in idiopathic pulmonary fibrosis also reported no significant change in reduced $\mathrm{PaO}_{2}$ at rest after a rehabilitation program [14]. Thus, it still remains difficult to distinguish parameters concerning the training outcome of induced oxygen desaturation in advance of rehabilitation intervention.

In conclusion, the effectiveness of exercise training on exercise-induced oxygen desaturation was not found in more than half of patients with systemic sclerosis despite improvements in exercise capacity. Even though some patients showed improvements in both walk distance and $\Delta \mathrm{SpO}_{2}$, the mechanism of amelioration in exercise-induced oxygen desaturation should be proven in the future.

\section{REFERENCES}

[1] Almeida, I., Faria, R., Vita, P. and Vasconcelos, C. (2011) Systemic sclerosis refractory disease: From the skin to the heart. Autoimmunity Reviews, 10, 693-701. doi:10.1016/j.autrev.2011.04.025

[2] Oliveira, N.C., dos Santos Sabbag, L.M., de Sa Pinto, A.L., Borges, C.L. and Lima, F.R. (2009) Aerobic exercise is safe and effective in systemic sclerosis. International Journal of Sports Medicine, 30, 728-732. doi:10.1055/s-0029-1224180

[3] Garin, M.C., Highland, K.B., Silver, R.M. and Strange, C. (2009) Limitations to the 6-minute walk test in interstitial lung disease and pulmonary hypertension in scleroderma. The Journal of Rheumatology, 36, 330-336. doi:10.3899/jrheum.080447

[4] Villalba, W.O., Sampaio-Barros, P.D., Pereira, M.C., Cerqueira, E.M.F.P., Leme Jr., C.A., Marques-Neto, J.F., et al. (2007) Six-minute walk test for the evaluation of pulmonary disease severity in scleroderma patients. Chest, 131,

\section{7-222. doi:10.1378/chest.06-0630}

[5] Cuomo, G., Santoriello, C., Polverino, F., Ruocco, L., Valentini, G. and Polverino, M. (2010) Impaired exercise performance in systemic sclerosis and its clinical correlations. Scandinavian Journal of Rheumatology, 39, 330335. doi:10.3109/03009740903555358

[6] Plazak, W., Gryga, K., Sznajd, J., Tomkiewicz-Pajak, L., Suchon, E., Wilisowska, J., et al. (2011) Diastolic heart dysfunction, increased pulmonary capillary wedge pressure and impaired exercise tolerance in patients with systemic sclerosis. Kardiologia Polska, 69, 243-249.

[7] Akdogan, A., Kaya, E.B. Sahin, A., Okutucu, S., Yakut, E., Kalyoncu, U., et al. (2011) Relationship between left ventricular diastolic dysfunction and six minute walk test in patients with systemic sclerosis. International Journal of Rheumatic Diseases, 14, 379-383. doi:10.1111/j.1756-185X.2011.01672.x

[8] Ferreira, A., Garvey, C., Connors, G.L., Hilling, L., Rigler, J., Farrell, S., et al. (2009) Pulmonary rehabilitation in interstitial lung disease: Benefits and predictors of response. Chest, 135, 442-447. doi:10.1378/chest.08-1458

[9] Mugii, N., Someya, F. and Hasegawa, M. (2011) Reduced hypoxia risk in a systemic sclerosis patient with interstitial lung disease after long-term pulmonary rehabilitation. Clinical Medicine Insights: Case Reports, 4, 53-56.

[10] Enright, P.L. and Sherrill, D.L. (1998) Reference equations for the six-minute walk in healthy adults. American Journal of Respiratory and Critical Care, 158, 1384-1387. doi:10.1164/ajrccm.158.5.9710086

[11] Wilsher, M., Good, N., Hopkins, R., Young, P., Milne, D., Gibson, A., et al. (2012) The six-minute walk test using forehead oximetry is reliable in the assessment of scleroderma lung disease. Respirology, 17, 647-652. doi:10.1111/j.1440-1843.2012.02133.x

[12] Spruit, M.A., Janssen, D.J.A., Franssen, F.M.E. and Wouters, E.F.M. (2009) Rehabilitation and palliative care in lung fibrosis. Respirology, 14, 781-787. doi:10.1111/j.1440-1843.2009.01599.x

[13] Wemeau-Stervinou, L., Perez, T., Murphy, C., Polge, A.-S. and Wallaert, B. (2012) Lung capillary blood volume and membrane diffusion in idiopathic interstitial pneumonia. Respiratory Medicine, 106, 564-570. doi:10.1016/j.rmed.2011.12.011

[14] Nishiyama, O., Kondoh, Y., Kimura, T., Kato, K., Kataoka, K., Ogawa, T., et al. (2008) Effects of pulmonary rehabilitation in patients with idiopathic pulmonary fibrosis. Respirology, 13, 394-399. doi:10.1111/j.1440-1843.2007.01205.x

[15] Handa, R., Gupta, K., Malhotra, A., Jain, P., Kamath, P.K., Aggarwal, P., et al. (1999) Cardiac involvement in limited systemic sclerosis: Non-invasive assessment in asymptomatic patients. Clinical Rheumatology, 18, 136-139. doi:10.1007/s100670050071

[16] Poanta, L., Dadu, R., Tiboc, C., Rednic, S. and Dumitrascu, D. (2009) Systolic and diastolic function in patients with systemic sclerosis. European Journal of Internal Medicine, 20, 378-382. doi:10.1016/j.ejim.2008.10.011

[17] Rosato, E., Maione, S., Vitarelli, A., Giunta, A., Fontanella, L., de Horatio, L.T., et al. (2009) Regional diastolic 
function by tissue Doppler echocardiography in systemic sclerosis: correlation with clinical variables. Rheumatology International, 29, 913-919. doi:10.1007/s00296-008-0827-X

[18] Lee, S-W., Choi, E.-Y., Jung, S.Y., Choi, S.T., Lee, S.-K. and Park and Y.-B. (2010) E/E' ratio is more sensitive than $\mathrm{E} / \mathrm{A}$ ratio for detection of left ventricular diastolic dysfunction in patients with systemic sclerosis. Clinical and Experimental Rheumatology, 28, S12-S17.

[19] Allanore, Y. and Meune, C. (2010) Primary myocardial involvement in systemic sclerosis: Evidence for a microvascular origin. Clinical and Experimental Rheumatology, 28, S48-S53.

[20] Alkotob, M.L., Soltani, P., Sheatt, M.A., Katsetos, M.C., Rothfield, N., Hager, D., et al. (2006) Reduced exercise capacity and stress-induced pulmonary hypertension in patients with scleroderma. Chest, 130, 176-181.

\section{doi:10.1378/chest.130.1.176}

[21] Koh, E.T., Lee, P., Gladman, D.D. and Abu-Shakra, M. (1996) Pulmonary hypertension in systemic sclerosis: An analysis of 17 patients. British Journal of Rheumatology, 35, 989-993. doi:10.1093/rheumatology/35.10.989

[22] D’Alto, M., Ghio, S., D'Andrea, A., Pazzano, A.S., Argiento, P., Camporotondo, R., et al. (2011) Inappropriate exercise-induced increase in pulmonary artery pressure in patients with systemic sclerosis. Heart, 97, 112-117. doi:10.1136/hrt.2010.203471

[23] Fernandes, F., Ramires, F.J.A., Arteaga, E., Ianni, B.A., Bonfa, E.S.D.O. and Mady, C. (2003) Cardiac remodeling in patients with systemic sclerosis with no signs or symptoms of heart failure: An endomyocardial biopsy study. Journal of Cardiac Failure, 9, 311-317. doi:10.1054/jcaf.2003.51 